\title{
Sasa-Satsuma hierarchy of integrable evolution equations
}

U. Bandelow, A. Ankiewicz, Sh. Amiranashvili, and N. Akhmediev

Citation: Chaos 28, 053108 (2018); doi: 10.1063/1.5030604

View online: https://doi.org/10.1063/1.5030604

View Table of Contents: http://aip.scitation.org/toc/cha/28/5

Published by the American Institute of Physics

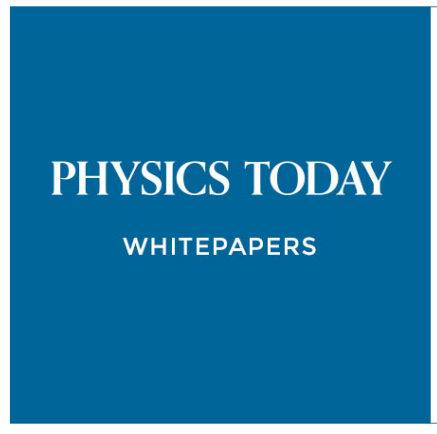

ADVANCES IN PRECISION MOTION CONTROL

Piezo Flexure Mechanisms and Air Bearings
READ NOW

PRESENTED BY

PI 


\title{
Sasa-Satsuma hierarchy of integrable evolution equations
}

\author{
U. Bandelow, ${ }^{1, a)}$ A. Ankiewicz, ${ }^{2}$ Sh. Amiranashvili, ${ }^{1}$ and N. Akhmediev ${ }^{2}$ \\ ${ }^{1}$ Weierstrass Institute for Applied Analysis and Stochastics, Mohrenstraße 39, 10117 Berlin, Germany \\ ${ }^{2}$ Optical Sciences Group, Research School of Physics and Engineering, The Australian National University, \\ Canberra, ACT 2600, Australia
}

(Received 23 March 2018; accepted 26 April 2018; published online 17 May 2018)

\begin{abstract}
We present the infinite hierarchy of Sasa-Satsuma evolution equations. The corresponding Lax pairs are given, thus proving its integrability. The lowest order member of this hierarchy is the nonlinear Schrödinger equation, while the next one is the Sasa-Satsuma equation that includes thirdorder terms. Up to sixth-order terms of the hierarchy are given in explicit form, while the provided recurrence relation allows one to explicitly write all higher-order terms. The whole hierarchy can be combined into a single general equation. Each term in this equation contains a real independent coefficient that provides the possibility of adapting the equation to practical needs. A few examples of exact solutions of this general equation with an infinite number of terms are also given explicitly. Published by AIP Publishing. https://doi.org/10.1063/1.5030604
\end{abstract}

The Sasa-Satsuma equation (SSE) belongs to a family of integrable evolution equations that is known to have soliton and rogue wave solutions. It is an extension of the well-known nonlinear Schrödinger equation that has been supplemented with terms responsible for thirdorder dispersion and higher-order nonlinear effects. The additional terms in this extension are chosen in a specific way, in order to ensure integrability of the resulting evolution equation. This extension is unique and also wellknown.

However, what is presently unknown is the fact that the SSE can be further extended with additional higherorder terms in such a way that integrability of the resulting more general equation is still retained. Here, we show, for the first time, that such a generalized SSE can have an infinite number of higher-order terms and that this more general equation is also integrable. Moreover, the additions contain free real parameters that can make the generalized SSE a versatile tool for the study of solitons and rogue waves. We present basic solutions for the solitary and rogue waves for this equation with an infinite number of terms and for any combination of the free parameters. We also explain why the generalized fourthorder Sasa-Satsuma equation was missed in the previous literature.

\section{INTRODUCTION}

The nonlinear Schrödinger equation (NLSE) is one of the basic models of nonlinear wave propagation in optical fibers, ${ }^{1}$ water waves, ${ }^{2,3}$ and generally in nonlinear dispersive media. ${ }^{4,5}$ This equation and its variations have been instrumental in describing phenomena of temporal and spatial soliton propagation, ${ }^{5}$ their interactions, ${ }^{6}$ modulation instability, ${ }^{7}$ periodic and localized breathers, ${ }^{8-11}$ supercontinuum radiation, ${ }^{12}$ Fermi-Pasta-Ulam Recurrence, ${ }^{13}$ Bose-Einstein condensates, ${ }^{14-16}$ and rogue waves. ${ }^{17-22}$ While being universal

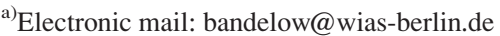

in covering such a diverse range of phenomena in physics, this equation is not a panacea for all problems. Though it correctly describes the basic features of wave dynamics, the finer details may be left unexplained when using the equation in its original form. In order to increase the accuracy of modelling, the NLSE has to be extended to include additional terms ${ }^{23}$ that are responsible for higher-order disper$\operatorname{sion}^{24}$ and nonlinear effects such as self-steepening and self-frequency shift. ${ }^{25}$ These terms are important in the description of higher-amplitude waves ${ }^{26,27}$ and shorter duration pulses. ${ }^{28}$

However, dealing with modified equations, while gaining in accuracy, we lose in terms of simplicity and integrability of the NLSE. The main problem with the NLSE extensions is the loss of integrability when the coefficients of additional terms are arbitrary. Then, the initial value problem cannot be solved analytically and each case requires numerical modelling. Fortunately, integrability is restored for special choices of the coefficients in the higher-order terms. For extensions including third-order terms, the choice of the coefficients that admit integrability is well-known. These cases include the Hirota ${ }^{29}$ equation and Sasa-Satsuma equation (SSE) ${ }^{30}$ However, the next step of such extensions is still not completely classified. For the branch of extensions that includes the Hirota equation, higher-order evolution equations are known. These include the fourth-order Lakshmanan-Porsezian-Daniel (LPD) equation ${ }^{31}$ and a fifthorder equation. ${ }^{32}$ Moreover, the whole infinite hierarchy of extensions and their soliton and rogue wave solutions have been presented explicitly in Refs. 33 and 34. However, for the branch of higher-order equations that include the SSE as a particular case, the fourth- and other higher-order extensions are presently unknown. We fill this gap in current knowledge of higher-order integrable equations in the present work.

Higher-order equations not only serve for improving the accuracy of the NLSE. They are independently important for a description of other physical phenomena such as the 
dynamics of the Heisenberg spin chain. ${ }^{35}$ Each integrable evolution equation is not just a special isolated case or a mathematical curiosity. Solutions can be analytically presented around the integrable cases in approximate forms, thus extending the range of their applicability. ${ }^{36}$ Thus, adding new equations to the family of integrable equations should be considered as adding significantly more power to our ability to do accurate mathematical modelling of physical phenomena.

To be specific, the major step forward made in the present work is presenting a new infinite hierarchy of evolution equations in the form

$$
\begin{aligned}
& i \psi_{t}+\alpha_{2} S_{2}[\psi(x, t)]-i \alpha_{3} S_{3}[\psi(x, t)]+\alpha_{4} S_{4}[\psi(x, t)] \\
& \quad-i \alpha_{5} S_{5}[\psi(x, t)]+\alpha_{6} S_{6}[\psi(x, t)]-i \alpha_{7} S_{7}[\psi(x, t)] \\
& +\alpha_{8} S_{8}[\psi(x, t)]-\cdots=0,
\end{aligned}
$$

where $S_{j}$ are the functionals of the order $j$ for the envelope function $\psi(x, t)$ and $\alpha_{j}$ are the arbitrary real coefficients. All functionals $S_{j}$ are given below in explicit form and we stress that the coefficients $\alpha_{j}$ are not small parameters. They are finite real numbers, thus making our approach far from being just another perturbation analysis. The allowance of variability of the coefficients $\alpha_{j}$ makes the hierarchy of Eq. (1) an infinitely variable integrable evolution equation for a variety of applications that describe soliton and rogue wave phenomena. It includes, as particular cases, the NLSE, mKdV, and SSE, thus linking the hierarchy (1) to these well known evolution equations.

More specifically, the lowest order functional $S_{2}[\psi(x, t)]$ in Eq. (1) is given by

$$
S_{2}[\psi(x, t)]=\psi_{x x}+4|\psi|^{2} \psi,
$$

while

$$
S_{3}[\psi(x, t)]=\psi_{x x x}+3\left(|\psi|^{2}\right)_{x} \psi+6|\psi|^{2} \psi_{x} .
$$

Thus, when all $\alpha_{j}$ are zero except for the $\alpha_{2}$, the hierarchy (1) is simply the NLSE. When, further, $\alpha_{3}$ is nonzero and $\alpha_{2}=\frac{1}{2}$, we have the SSE

$$
i \psi_{t}+\frac{\psi_{x x}}{2}+2|\psi|^{2} \psi=i \alpha_{3}\left[\psi_{x x x}+3\left(|\psi|^{2}\right)_{x} \psi+6|\psi|^{2} \psi_{x}\right] .
$$

All further extensions from fourth-order and higher and the recurrent relations for obtaining them are presented in this work. In particular, we show that

$$
\begin{aligned}
S_{4}[\psi(x, t)]= & \psi_{x x x x}+6 \bar{\psi}_{x x} \psi^{2}+24|\psi|^{4} \psi \\
& +12\left|\psi_{x}\right|^{2} \psi+14|\psi|^{2} \psi_{x x}+8 \bar{\psi} \psi_{x}^{2},
\end{aligned}
$$

and

$$
\begin{aligned}
S_{5}[\psi(x, t)]= & \psi_{x x x x x}+80|\psi|^{4} \psi_{x}+5 \psi^{2} \bar{\psi}_{x x x} \\
& +25 \psi\left(\left|\psi_{x}\right|^{2}\right)_{x}+40|\psi|^{2} \psi^{2} \bar{\psi}_{x} \\
& +20\left|\psi_{x}\right|^{2} \psi_{x}+15|\psi|^{2} \psi_{x x x}+30 \bar{\psi} \psi_{x} \psi_{x x},
\end{aligned}
$$

where $\bar{\psi}$ denotes the complex conjugate of $\psi$, and

$$
\begin{aligned}
S_{6}[\psi(x, t)]= & \psi_{x x x x x x}+55 \psi^{3}\left(\bar{\psi}_{x}\right)^{2}+45 \psi_{x}^{2} \bar{\psi}_{x x}+32 \psi \psi_{x} \bar{\psi}_{x x x} \\
& +43 \bar{\psi} \psi_{x} \psi_{x x x}+37 \psi \bar{\psi}_{x} \psi_{x x x}+175|\psi|^{2} \bar{\psi} \psi_{x}^{2} \\
& +53\left|\psi_{x x}\right|^{2} \psi+31 \bar{\psi} \psi_{x x}^{2}+20|\psi|^{2} \psi_{x x x x} \\
& +160|\psi|^{6} \psi+110 \bar{\psi} \psi^{3} \bar{\psi}_{x x}+330\left|\psi \psi_{x}\right|^{2} \psi \\
& +170|\psi|^{4} \psi_{x x}+8 \psi^{2} \bar{\psi}_{x x x x}+95\left|\psi_{x}\right|^{2} \psi_{x x}
\end{aligned}
$$

The expressions for $S_{7}[\psi(x, t)]$ and higher are too cumbersome to be given here, but our technique is straightforward, allowing one to write them explicitly for any order $j$. These expressions are different from those for the NLSE hierarchy given in Ref. 34. They comprise a different hierarchy of integrable equations. The reason is that the Lax pairs for these equations involve $3 \times 3$ matrices rather than $2 \times 2$ for the Hirota branch. As a result, the solutions of the SSE hierarchy are significantly more involved than those found in Ref. 34. Such complexity starts right from the lowest order equation of the hierarchy which is the SSE. ${ }^{30,37-42}$ As a result of this complexity, the solutions of the SSE are also highly nontrivial. Both soliton solutions ${ }^{37-39}$ and rogue wave solutions $^{43}$ have much more complicated structures than the corresponding solutions for the NLSE or Hirota equations. They involve more parameters in the solutions that allow us to describe more complicated profiles. Due to this complexity, only first-order solutions have been derived so far. Also due to this complexity, the SSE is an equation for which the higher-order hierarchy was unknown until now.

Physically, the difference between the two models seems to have its origin in the fact that phase and group velocities of waves in the case of the SSE do not coincide, ${ }^{44}$ which significantly distorts the shape of the solitons in comparison with the NLSE solutions. In particular, the SSE has single-soliton solutions that have no analogs in the NLSE case. In addition to the common bell-shaped solitons, it has soliton solutions with two maxima ${ }^{30}$ and even with multiple maxima. ${ }^{39}$ Moreover, the SSE has soliton solutions with complex oscillating patterns in the $(x, t)$-plane. ${ }^{38}$ Solutions become even more complicated when they contain a background in the form of a plane wave. ${ }^{40}$ Clearly, these complexities accumulate when dealing with the higher-order equations of the SSE hierarchy.

\section{LAX-PAIR FORMULATION}

Nonlinear evolution equations that arise from various branches of wave physics are formally integrable if they admit the zero-curvature representation

$$
U_{t}-V_{x}+[U, V]=0 .
$$

Given a properly chosen isospectral problem

$$
\phi_{x}=U(\lambda) \phi,
$$

with $\lambda$ being the spectral parameter for which $\lambda_{t}=0$, one can relate it to a hierarchy of nonlinear evolution equations

$$
U_{t}-V_{x}^{n}+\left[U, V^{n}\right]=0 .
$$

In this context, the choice of $U$ serves as a seed, from which the hierarchy $\left\{V^{n}\right\}$ can be derived. For each stage $n$ of the 
hierarchy, there is an independent Hamiltonian flow given by

$$
U_{t_{n}}=V_{x}^{n}-\left[U, V^{n}\right] .
$$

Here, we are specifically interested in the hierarchy associated with the Sasa-Satsuma equation, where $U$ and $V$ are $3 \times 3$ matrices. Note that the hierarchy of Manakov equations

$$
\begin{aligned}
& i u_{t}+u_{x x}+2\left(|u|^{2}+|v|^{2}\right) u=0, \\
& i v_{t}+v_{x x}+2\left(|u|^{2}+|v|^{2}\right) v=0,
\end{aligned}
$$

which requires the $3 \times 3$ matrices, ${ }^{45}$ can also be considered in the same way. ${ }^{46}$ This set of equations is associated with the following $3 \times 3$ matrix spectral problem:

$$
\phi_{x}=U \phi, \quad \phi=\left(\begin{array}{c}
\phi_{1} \\
\phi_{2} \\
\phi_{3}
\end{array}\right), \quad U=\left(\begin{array}{ccc}
-\lambda & u & w \\
v & \lambda & 0 \\
r & 0 & \lambda
\end{array}\right) .
$$

This formulation is rather general and admits two special reductions. One of them is the coupled modified Korteweg-de Vries hierarchy which corresponds to the choice of $v=u$ and $r=w$. Another one is the Sasa-Satsuma hierarchy with the choice of $w=v$ and $r=u$. This latter case is addressed in the present work.

Thus, given the spectral problem (13), we seek a hierarchy $V^{n}$ which solves Eq. (11). In order to find it, we expand the matrix elements of $V^{n}$ in a polynomial

$$
V_{i j}^{n}=\sum_{k=0}^{n} v_{i j}^{k} \lambda^{n-k}, \quad \text { with } \quad v_{i j}^{k}=v_{i j}^{k}(x, t)
$$

and $V_{11}=-\left(V_{22}+V_{33}\right)$. Inserting (14) into (10), we obtain, for $n=1$

$$
\begin{aligned}
& \left(\begin{array}{ccc}
0 & u_{t} & w_{t} \\
v_{t} & 0 & 0 \\
r_{t} & 0 & 0
\end{array}\right)-\partial_{x}\left(\begin{array}{ccc}
v_{11}^{1} & v_{12}^{1} & v_{13}^{1} \\
v_{21}^{1} & v_{22}^{1} & v_{23}^{1} \\
v_{31}^{1} & v_{32}^{1} & v_{33}^{1}
\end{array}\right)-\lambda \partial_{x}\left(\begin{array}{ccc}
v_{11}^{0} & v_{12}^{0} & v_{13}^{0} \\
v_{21}^{0} & v_{22}^{0} & v_{23}^{0} \\
v_{31}^{0} & v_{32}^{0} & v_{33}^{0}
\end{array}\right)-2 \lambda^{2}\left(\begin{array}{ccc}
0 & v_{12}^{0} & v_{13}^{0} \\
-v_{21}^{0} & 0 & 0 \\
-v_{31}^{0} & 0 & 0
\end{array}\right) \\
& +\lambda\left(\begin{array}{ccc}
u v_{21}^{0}+v_{31}^{0} w-v v_{12}^{0}-r v_{13}^{0} & u\left(2 v_{22}^{0}+v_{33}^{0}\right)+v_{32}^{0} w-2 v_{12}^{1} & u v_{23}^{0}-2 v_{13}^{1}+\left(v_{22}^{0}+2 v_{33}^{0}\right) w \\
2 v_{21}^{1}-r v_{23}^{0}-v\left(2 v_{22}^{0}+v_{33}^{0}\right) & v v_{12}^{0}-u v_{21}^{0} & v v_{13}^{0}-v_{21}^{0} w \\
2 v_{31}^{1}-v v_{32}^{0}-r\left(v_{22}^{0}+2 v_{33}^{0}\right) & r v_{12}^{0}-u v_{31}^{0} & r v_{13}^{0}-v_{31}^{0} w
\end{array}\right) \\
& +\left(\begin{array}{ccc}
u v_{21}^{1}+w v_{31}^{1}-v v_{12}^{1}-r w v_{13}^{1} & u\left(2 v_{22}^{1}+v_{33}^{1}\right)+w v_{32}^{1} & u v_{23}^{1}+w\left(v_{22}^{1}+2 v_{33}^{1}\right) \\
-v\left(2 v_{22}^{1}+v_{33}^{1}\right)-r v_{23}^{1} & v v_{12}^{1}-u v_{21}^{1} & v v_{13}^{1}-w v_{21}^{1} \\
-v v_{32}^{1}-r\left(v_{22}^{1}+2 v_{33}^{1}\right) & r v_{12}^{1}-u v_{31}^{1} & r v_{13}^{1}-w v_{31}^{1}
\end{array}\right)=0 .
\end{aligned}
$$

Apparently, the functions $v_{12}^{0}=v_{13}^{0}=v_{21}^{0}=v_{31}^{0}=0$ are zeros together with their derivatives. Then, the above system reduces to

$$
\begin{aligned}
& \left(\begin{array}{ccc}
0 & u_{t} & w_{t} \\
v_{t} & 0 & 0 \\
r_{t} & 0 & 0
\end{array}\right)-\partial_{x}\left(\begin{array}{ccc}
v_{11}^{1} & v_{12}^{1} & v_{13}^{1} \\
v_{21}^{1} & v_{22}^{1} & v_{23}^{1} \\
v_{31}^{1} & v_{32}^{1} & v_{33}^{1}
\end{array}\right)-\lambda \partial_{x}\left(\begin{array}{ccc}
v_{11}^{0} & 0 & 0 \\
0 & v_{22}^{0} & v_{23}^{0} \\
0 & v_{32}^{0} & v_{33}^{0}
\end{array}\right) \\
& +\lambda\left(\begin{array}{ccc}
0 & u\left(2 v_{22}^{0}+v_{33}^{0}\right)+v_{32}^{0} w-2 v_{12}^{1} & u v_{23}^{0}-2 v_{13}^{1}+\left(v_{22}^{0}+2 v_{33}^{0}\right) w \\
2 v_{21}^{1}-r v_{23}^{0}-v\left(2 v_{22}^{0}+v_{33}^{0}\right) & 0 & 0 \\
2 v_{31}^{1}-v v_{32}^{0}-r\left(v_{22}^{0}+2 v_{33}^{0}\right) & 0 & 0
\end{array}\right) \\
& +\left(\begin{array}{ccc}
u v_{21}^{1}+w v_{31}^{1}-v v_{12}^{1}-r w v_{13}^{1} & u\left(2 v_{22}^{1}+v_{33}^{1}\right)+w v_{32}^{1} & u v_{23}^{1}+w\left(v_{22}^{1}+2 v_{33}^{1}\right) \\
-v\left(2 v_{22}^{1}+v_{33}^{1}\right)-r v_{23}^{1} & v v_{12}^{1}-u v_{21}^{1} & v v_{13}^{1}-w v_{21}^{1} \\
-v v_{32}^{1}-r\left(v_{22}^{1}+2 v_{33}^{1}\right) & r v_{12}^{1}-u v_{31}^{1} & r v_{13}^{1}-w v_{31}^{1}
\end{array}\right)=0 .
\end{aligned}
$$

Moreover, the following elements $v_{23}^{0}=c_{1}, v_{32}^{0}=c_{2}, v_{22}^{0}=c_{3}, v_{33}^{0}=c_{4}$, and $v_{11}^{0}=-\left(c_{1}+c_{4}\right)$ are constants. Then, we have, in particular,

$$
\left(\begin{array}{ccc}
0 & u\left(2 c_{3}+c_{4}\right)+c_{2} w-2 v_{12}^{1} & u c_{1}-2 v_{13}^{1}+\left(c_{3}+2 c_{4}\right) w \\
2 v_{21}^{1}-r c_{1}-v\left(2 c_{3}+c_{4}\right) & 0 & 0 \\
2 v_{31}^{1}-v c_{2}-r\left(c_{3}+2 c_{4}\right) & 0 & 0
\end{array}\right)=0,
$$


and, as a consequence

$$
v_{12}^{1}=\frac{w c_{2}+u\left(2 c_{3}+c_{4}\right)}{2}, \quad v_{21}^{1}=\frac{r c_{1}+v\left(3 c_{3}+c_{4}\right)}{2}, \quad v_{13}^{1}=\frac{u c_{1}+w\left(c_{3}+2 c_{4}\right)}{2}, \quad v_{31}^{1}=\frac{v c_{2}+r\left(c_{3}+2 c_{4}\right)}{2} .
$$

In the next step, we solve the remaining equation

$$
\left(\begin{array}{ccc}
0 & u_{t} & w_{t} \\
v_{t} & 0 & 0 \\
r_{t} & 0 & 0
\end{array}\right)-\partial_{x}\left(\begin{array}{ccc}
v_{11}^{1} & v_{12}^{1} & v_{13}^{1} \\
v_{21}^{1} & v_{22}^{1} & v_{23}^{1} \\
v_{31}^{1} & v_{32}^{1} & v_{33}^{1}
\end{array}\right)+\left(\begin{array}{ccc}
-\left(v_{22}^{1}+v_{33}^{1}\right) & u\left(2 v_{22}^{1}+v_{33}^{1}\right)+w v_{32}^{1} & u v_{23}^{1}+w\left(v_{22}^{1}+2 v_{33}^{1}\right) \\
-v\left(2 v_{22}^{1}+v_{33}^{1}\right)-r v_{23}^{1} & v v_{12}^{1}-u v_{21}^{1} & v v_{13}^{1}-w v_{21}^{1} \\
-v v_{32}^{1}-r\left(v_{22}^{1}+2 v_{33}^{1}\right) & r v_{12}^{1}-u v_{31}^{1} & r v_{13}^{1}-w v_{31}^{1}
\end{array}\right)=0
$$

for $v_{23}^{1}, v_{32}^{1}, v_{22}^{1}, v_{33}^{1}$, which gives

$$
v_{23}^{1}=\partial^{-1}\left(v v_{13}^{1}-w v_{21}^{1}\right), \quad v_{32}^{1}=\partial^{-1}\left(r v_{12}^{1}-u v_{31}^{1}\right), \quad v_{22}^{1}=\partial^{-1}\left(v v_{12}^{1}-u v_{21}^{1}\right), \quad v_{33}^{1}=\partial^{-1}\left(r v_{13}^{1}-w v_{31}^{1}\right) .
$$

For brevity, we have introduced the notation $\partial=\partial_{x}$ and the corresponding integral operator $\partial^{-1}$, such that $\partial \partial^{-1}=\partial^{-1} \partial=1$. The 4 remaining equations are the desired integro-differential equations for $u, v, r, w$ in the lowest rank of the infinite Manakov hierarchy

$$
\begin{gathered}
u_{t}=c_{2} w_{x}+\left(2 c_{3}+c_{4}\right) u_{x}+w \partial^{-1}\left[c_{2} u v-r\left(c_{2} w+\left(c_{3}-c_{4}\right) u\right)\right]+u \partial^{-1}\left(c_{1} r u-c_{2} v w\right), \\
w_{t}=c_{1} u_{x}+\left(c_{3}+2 c_{4}\right) w_{x}-u \partial^{-1}\left[c_{1} u v-w\left(c_{1} r+\left(c_{3}-c_{4}\right) v\right)\right]+w \partial^{-1}\left(c_{2} v w-c_{1} r u\right), \\
v_{t}=c_{1} r_{x}+\left(2 c_{3}+c_{4}\right) v_{x}+r \partial^{-1}\left[c_{1} u v-w\left(c_{1} r+\left(c_{3}-c_{4}\right) v\right)\right]+v \partial^{-1}\left(c_{2} v w-c_{1} r u\right), \\
r_{t}=c_{2} v_{x}+\left(c_{3}+2 c_{4}\right) r_{x}-v \partial^{-1}\left[c_{2} u v-r\left(c_{2} w+\left(c_{3}-c_{4}\right) u\right)\right]+r \partial^{-1}\left(c_{1} r u-c_{2} v w\right) .
\end{gathered}
$$

Based on this set of equations, we can find the coefficients $v_{i j}^{n}(x, t)$ and the desired integro-differential equations relative to $u, v, r$, and $w$. This can be done step-by-step for the higher orders $n>1$. This procedure would be straightforward but becomes increasingly involved as $n$ increases. However, there are ways to simplify the derivations. In order to illustrate the idea, let us start with the Manakov case.

\section{RECURSIVE RELATIONS FOR CONSTRUCTION OF THE HIERARCHY}

Our straightforward method can be cast in a more compact notation. Given the spectral problem (13), we seek a hierarchy $V^{n}$ which solves Eq. (11). The problem (11) can be cast as a problem for two 4 -vectors, $G$ and $H$, relative to four independent functions $u, v, w$, and $r^{46}$

$$
H=\left(\begin{array}{l}
V_{12} \\
V_{21} \\
V_{13} \\
V_{31}
\end{array}\right)=\left(\begin{array}{l}
H_{1} \\
H_{2} \\
H_{3} \\
H_{4}
\end{array}\right), \quad G=\left(\begin{array}{l}
V_{23} \\
V_{32} \\
V_{22} \\
V_{33}
\end{array}\right)=\left(\begin{array}{l}
G_{1} \\
G_{2} \\
G_{3} \\
G_{4}
\end{array}\right) .
$$

We keep in mind that

$$
\operatorname{Tr}[V]=0, \quad \text { i.e. } \quad V_{11}=-\left(V_{22}+V_{33}\right) .
$$

Let us also define 3 matrices

$$
\begin{aligned}
K_{1} & =\left(\begin{array}{cccc}
0 & -w & -2 u & -u \\
r & 0 & 2 v & v \\
-u & 0 & -w & -2 w \\
0 & v & r & 2 r
\end{array}\right), \\
K_{2} & =\left(\begin{array}{cccc}
0 & w & -v & 0 \\
-r & 0 & 0 & u \\
-v & u & 0 & 0 \\
0 & 0 & -r & w
\end{array}\right) \\
J & =2\left(\begin{array}{cccc}
-1 & 0 & 0 & 0 \\
0 & 1 & 0 & 0 \\
0 & 0 & -1 & 0 \\
0 & 0 & 0 & 1
\end{array}\right)
\end{aligned}
$$

with

$$
J^{-1}=\frac{1}{4} J
$$

Then, the set of equations for $H$ and $G$ are

$$
\begin{gathered}
\partial H+K_{1} G=\lambda J H, \\
\partial G+K_{2} H=0 .
\end{gathered}
$$

Together with $\partial_{t} U=\lambda J H$, this set is equivalent to (11). ${ }^{46}$ As before, we expand the matrix elements of $V^{n}$ in a polynomial 
(14) which allows us to present the 4-vectors, $G$ and $H$, in the following form:

$$
H=\sum_{k=0}^{n} h^{k} \lambda^{n-k}, \quad G=\sum_{k=0}^{n} g^{k} \lambda^{n-k},
$$

with the 4-vectors, $h^{k}=h^{k}(x, t)$ and $g^{k}=g^{k}(x, t)$

$$
\begin{aligned}
& h^{k}=\left(\begin{array}{l}
h_{1}^{k} \\
h_{2}^{k} \\
h_{3}^{k} \\
h_{4}^{k}
\end{array}\right)=\left(\begin{array}{c}
v_{12}^{k}(x, t) \\
v_{21}^{k}(x, t) \\
v_{13}^{k}(x, t) \\
v_{31}^{k}(x, t)
\end{array}\right), \\
& g^{k}=\left(\begin{array}{l}
g_{1}^{k} \\
g_{2}^{k} \\
g_{3}^{k} \\
g_{4}^{k}
\end{array}\right)=\left(\begin{array}{c}
v_{23}^{k}(x, t) \\
v_{32}^{k}(x, t) \\
v_{22}^{k}(x, t) \\
v_{33}^{k}(x, t)
\end{array}\right) .
\end{aligned}
$$

For $n=1$, we get from (22) and (23)

$$
\begin{gathered}
\partial h^{1}+K_{1} g^{1}+\lambda\left(\partial h^{0}+K_{1} g^{0}-J h^{1}\right)=\lambda^{2} J h^{0} \\
\lambda\left(\partial g^{0}+K_{2} h^{0}\right)+\left(\partial g^{1}+K_{2} h^{1}\right)=0 .
\end{gathered}
$$

It follows, from the r.h.s. of (27) that $h^{0}=0$, and hence $\partial h^{0}$ $=0$. With this condition, we get from (28) that $\partial g^{0}=0$. Consequently, $g^{0}=\left(c_{1}, c_{2}, c_{3}, c_{4}\right)^{\mathrm{T}}=$ const. In turn, we get from (27) that $h^{1}=J^{-1} K_{1} g^{0}$, and, again, from (28) $g^{1}$ $=-\partial^{-1} K_{2} h^{1}$. The remaining equation

$$
\begin{aligned}
\partial_{t_{0}}(u, v, w, r)^{\mathrm{T}} & =\partial h^{1}+K_{1} g^{1} \\
& =\partial J^{-1} K_{1} g^{0}-K_{1} \partial^{-1} K_{2} J^{-1} K_{1} g^{0}
\end{aligned}
$$

is then the lowest order equation in the Manakov hierarchy. Writing each of the equations individually, we arrive to exactly the same set as (17-20).

All higher orders $n>1$ can be obtained successively by the following procedure. First, from (22), $\propto \lambda: \partial h^{n}+K_{1} g^{n}$ $=J h^{n+1}$, we advance $h$ one step in $n$

$$
h^{n+1}=J^{-1}\left(\partial h^{n}+K_{1} g^{n}\right) .
$$

Second, from (23), $\partial g^{n+1}+K_{2} h^{n+1}=0$, we advance one step in $g$

$$
g^{n+1}=-\partial^{-1}\left(K_{2} h^{n+1}\right) .
$$

This determines the remaining coefficients of the next order $(n+1)$. Finally, with these coefficients, we obtain from (22), $\propto \lambda^{0}$ the equations for the next order $(n+1)$ of the Manakov hierarchy

$$
\partial_{t_{n}}(u, v, w, r)^{\mathrm{T}}=\partial h^{n+1}+K_{1} g^{n+1} .
$$

The validity of (32) can be checked using the compatibility condition (11).

Now, the r.h.s. of Eq. (32) can be used for obtaining the next order of $h$

$$
h^{n+2}=J^{-1}\left(\partial h^{n+1}+K_{1} g^{n+1}\right),
$$

which is the same as Eq. (30) for $n \rightarrow n+1$. The procedure can be repeated indefinitely. This recursive scheme creates the infinite hierarchy of integrable equations for the general Manakov system.

With this general idea, we can reformulate the hierarchy in an even more compact form. Let us denote

$$
Q^{n}=\partial h^{n+1}+K_{1} g^{n+1}
$$

such that Eq. (32) becomes

$$
\partial_{t_{n}}(u, v, w, r)^{\mathrm{T}}=Q^{n} .
$$

Expressions for the next order then become

$$
h^{n+1}=J^{-1} Q^{n}, \quad g^{n+1}=-\partial^{-1}\left(K_{2} J^{-1} Q^{n}\right),
$$

and, finally, the closed form of the recursive expression is

$$
Q^{n+1}=\partial\left(J^{-1} Q^{n}\right)-K_{1} \partial^{-1}\left(K_{2} J^{-1} Q^{n}\right) .
$$

This allows us to calculate directly the desired integrodifferential equations $Q^{n+1}$ for the next level of hierarchy from the previous one. As it can be seen from Eq. (37), at each iteration, the first term in the r.h.s. increases the order of derivatives by one, whereas the second term increases the order of nonlinearity by two. This general rule allows us to predict the form of the higher-order terms for any $n$.

It would be instructive to write in detail the recursive equations for the second order. Namely, for the order $n=2$, we have

$$
\begin{gathered}
u_{t}=-\frac{w \int_{1}^{x} \mathcal{I}_{1}^{1} d z+\int_{1}^{x} \mathcal{I}_{2}^{1} d z+2 u \int_{1}^{x} \mathcal{I}_{3}^{1} d z+\mathcal{U}}{4}, \\
w_{t}=-\frac{w \int_{1}^{x} \mathcal{I}_{1}^{2} d z+2 w \int_{1}^{x} \mathcal{I}_{2}^{2} d z+u \int_{1}^{x} \mathcal{I}_{3}^{2} d z+\mathcal{W}}{4}, \\
v_{t}=\frac{v \int_{1}^{x} \mathcal{I}_{1}^{3} d z+2 v \int_{1}^{x} \mathcal{I}_{2}^{3} d z+r \int_{1}^{x} \mathcal{I}_{3}^{3} d z+\mathcal{V}}{4}, \\
r_{t}=\frac{v \int_{1}^{x} \mathcal{I}_{1}^{4} d z+r \int_{1}^{x} \mathcal{I}_{2}^{4} d z+2 r \int_{1}^{x} \mathcal{I}_{3}^{4} d z+\mathcal{R}}{4},
\end{gathered}
$$

where

$$
\begin{aligned}
& \mathcal{I}_{1}^{1}=r\left(2 u\left(-2 F_{1}(z)+c_{7}-c_{8}\right)+2 w F_{2}(z)-c_{2} w_{z}-\left(2 c_{3}+c_{4}\right) u_{z}\right)-u\left(2 v F_{2}(z)+c_{2} v_{z}+\left(c_{3}+2 c_{4}\right) r_{z}\right), \\
& \mathcal{I}_{2}^{1}=r\left(2 u F_{3}(z)-c_{1} u_{z}-\left(c_{3}+2 c_{4}\right) w_{z}\right)-w\left(2 v F_{2}(z)+c_{2} v_{z}+\left(c_{3}+2 c_{4}\right) r_{z}\right), \\
& \mathcal{I}_{3}^{1}=v\left(2 w F_{2}(z)-c_{2} w_{z}-\left(2 c_{3}+c_{4}\right) u_{z}\right)-u\left(2 r F_{3}(z)+c_{1} r_{z}+\left(2 c_{3}+c_{4}\right) v_{z}\right),
\end{aligned}
$$




$$
\begin{aligned}
& \mathcal{I}_{1}^{2}=v\left(2 w F_{2}(z)-c_{2} w_{z}-\left(2 c_{3}+c_{4}\right) u_{z}\right)-u\left(2 r F_{3}(z)+c_{1} r_{z}+\left(2 c_{3}+c_{4}\right) v_{z}\right), \\
& \mathcal{I}_{2}^{2}=r\left(2 u F_{3}(z)-c_{1} u_{z}-\left(c_{3}+2 c_{4}\right) w_{z}\right)-w\left(2 v F_{2}(x)+c_{2} v_{z}+\left(c_{3}+2 c_{4}\right) r_{z}\right), \\
& \mathcal{I}_{3}^{2}=4 v w F_{1}(z)+2(u v-r w) F_{3}(z)-c_{1}\left(r_{z} w+u_{z} v\right)-\left(c_{3}+2 c_{4}\right) v w_{z}-\left(2 c_{3}+c_{4}\right) v_{z} w+2\left(c_{8}-c_{7}\right) v w, \\
& \mathcal{I}_{1}^{3}=r\left(2 u F_{3}(z)-c_{1} u_{z}-\left(c_{3}+2 c_{4}\right) w_{z}\right)-w\left(2 v F_{2}(z)+c_{2} v_{z}+\left(c_{3}+2 c_{4}\right) r_{z}\right), \\
& \mathcal{I}_{2}^{3}=v\left(2 w F_{2}(z)-c_{2} w_{z}-\left(2 c_{3}+c_{4}\right) u_{z}\right)-u\left(2 r F_{3}(z)+c_{1} r_{z}+\left(2 c_{3}+c_{4}\right) v_{z}\right), \\
& \mathcal{I}_{3}^{3}=4 v w F_{1}(z)+2(u v-r w) F_{3}(z)-c_{1}\left(r_{z} w+u_{z} v\right)-\left(c_{3}+2 c_{4}\right) v w_{z}-\left(2 c_{3}+c_{4}\right) v_{z} w+2\left(c_{10}-c_{7}\right) v w, \\
& \mathcal{I}_{1}^{4}=r\left(2 u\left(-2 F_{1}(z)+c_{7}-c_{10}\right)+2 w F_{2}(z)-c_{2} w_{z}-\left(2 c_{3}+c_{4}\right) u_{z}\right)-u\left(2 v F_{2}(z)+c_{2} v_{z}+\left(c_{3}+2 c_{4}\right) r_{z}\right), \\
& \mathcal{I}_{2}^{4}=v\left(2 w F_{2}(z)-c_{2} w_{z}-\left(2 c_{3}+c_{4}\right) u_{z}\right)-u\left(2 r F_{3}(z)+c_{1} r_{z}+\left(2 c_{3}+c_{4}\right) v_{z}\right), \\
& \mathcal{I}_{3}^{4}=r\left(2 u F_{3}(z)-c_{1} u_{z}-\left(c_{3}+2 c_{4}\right) w_{z}\right)-w\left(2 v F_{2}(z)+c_{2} v_{z}+\left(c_{3}+2 c_{4}\right) r_{z}\right), \\
& \mathcal{U}=4\left(2 c_{11}+c_{12}\right) u-c_{0} u r w+4 c_{10} w+2 u_{x}\left(F_{1}(x)-2 c_{7}-c_{8}\right)+c_{1} r u^{2}-2 w_{x} F_{2}(x)-c_{2} r w^{2}+c_{2} w_{x x}+\left(2 c_{3}+c_{4}\right) u_{x x}, \\
& \mathcal{W}=4\left(c_{11}+2 c_{12}\right) w+4 c_{9} u+c_{0} u v w-2 w_{x}\left(F_{1}(x)+c_{7}+2 c_{8}\right)-2 u_{x} F_{3}(x)+c_{1} u_{x x}-c_{1} u^{2} v+c_{2} v w^{2}+\left(c_{3}+2 c_{4}\right) w_{x x}, \\
& \mathcal{V}=8 c_{2} v^{2} w+4\left(2 c_{11}+c_{12}\right) v+4 c_{10} r-c_{0} v w r-2 v_{x}\left(F_{1}(x)-2 c_{7}-c_{10}\right)+2 r_{x} F_{3}(x)+c_{1} r_{x x}-c_{1} r^{2} w+\left(2 c_{3}+c_{4}\right) v_{x x}, \\
& \mathcal{R}=-4 c_{10} v+c_{0} r u v+4\left(c_{11}+2 c_{12}\right) r+2 r_{x}\left(F_{1}(x)+c_{7}+2 c_{10}\right)+c_{1} r^{2} u+2 v_{x} F_{2}(x)-c_{2} u v^{2}+c_{2} v_{x x}+\left(c_{3}+2 c_{4}\right) r_{x x} .
\end{aligned}
$$

The above set of equations contains, among others, the original Manakov system (12). In order to show this, we set $c_{3}=c_{4}=i \frac{4}{3}$, and all other constants $c_{i}=0$. As a result, we obtain

$$
\begin{aligned}
-i u_{t} & =-u_{x x}+2(u v+r w) u, \\
-i v_{t} & =v_{x x}-2(u v+r w) v, \\
-i w_{t} & =-w_{x x}+2(u v+r w) w, \\
-i r_{t} & =r_{x x}-2(u v+r w) r .
\end{aligned}
$$

Identifying then $v=\bar{u}$ and $r=\bar{w}$ and setting $x \rightarrow i x$, we obtain

$$
\begin{aligned}
& i u_{t}+u_{x x}+2\left(|u|^{2}+|w|^{2}\right) u=0, \\
& i w_{t}+w_{x x}+2\left(|u|^{2}+|w|^{2}\right) w=0,
\end{aligned}
$$

which coincides with the original Manakov system (12) after renaming $w(x, t)$ to $v(x, t)$.

The results for the next order $n=3$ include two special cases:

(1) The coupled modified Korteweg-de Vries equations

$$
\begin{aligned}
& u_{t}=-u_{x x x}+6 u^{2} u_{x}+3 v^{2} u_{x}+3 u v v_{x}, \\
& v_{t}=-v_{x x x}+6 v^{2} v_{x}+3 u^{2} v_{x}+3 u v u_{x} .
\end{aligned}
$$

This is obtained by choosing $v=u, r=w$, and setting $c_{3}=c_{4}=\frac{8}{3}$, and all other constants $c_{i}=0$.

(2) The Sasa-Satsuma equation is obtained by choosing $w=v$ and $r=u$. Namely, if we set $c_{3}=c_{4}=\frac{8}{3} \epsilon$, and all other constants $c_{i}=0$, we first obtain

$$
\begin{aligned}
& \partial_{t} u=\epsilon\left(-3 u\left(3 v u_{x}+u v_{x}\right)+u_{x x x}\right), \\
& \partial_{t} v=\epsilon\left(-3 v\left(3 u v_{x}+v u_{x}\right)+v_{x x x}\right),
\end{aligned}
$$

which can be rewritten as

$$
\begin{aligned}
& \partial_{t} u=\epsilon\left(-3 u(u v)_{x}-6 u v u_{x}+u_{x x x}\right), \\
& \partial_{t} v=\epsilon\left(-3 v(u v)_{x}-6 u v v_{x}+v_{x x x}\right) .
\end{aligned}
$$

If we replace now $t \rightarrow i t$ and $x \rightarrow i x$, set $v=\bar{u}$ and $\psi=u$, we get the Sasa-Satsuma equation in the complex modified KdV (cmKdV) form ${ }^{30,47}$

$$
\psi_{t}+\epsilon\left(\psi_{x x x}+6|\psi|^{2} \psi_{x}+3 \psi\left|\psi^{2}\right|_{x}\right)=0
$$

Setting $\epsilon=-\alpha_{3}$ gives the standard form of Eq. (3), i.e., $\psi_{t}-\alpha_{3} S_{3}=0$.

\section{SASA-SATSUMA HIERARCHY}

Now, we are in a position to obtain the whole SasaSatsuma hierarchy that starts with Eq. (39) by choosing $w=v$ and $r=u$ and using the techniques described in Sec. III.

\section{A. Level 1}

We set $c_{3}=c_{4}$, and $c_{1}=c_{2}=c_{5}=c_{6}=c_{7}=c_{8}=0$. For the Sasa-Satsuma equation, which appears on level 3, we choose $c_{4}=\frac{8}{3} \epsilon$. On level 1 , we obtain the uncoupled linear equations

$$
\partial_{t} u=4 \epsilon u_{x}, \quad \partial_{t} v=4 \epsilon v_{x} .
$$

\section{B. Level 2}

On level 2, we obtain the second order equations

$$
\begin{gathered}
\partial_{t} u=c_{10} v+3 c_{12} u+8 \epsilon(u v) u-2 \epsilon u_{x x}, \\
\partial_{t} v=-c_{10} u-3 c_{12} v-8 \epsilon(u v) v+2 \epsilon v_{x x},
\end{gathered}
$$

in addition to

$$
\begin{gathered}
-\partial_{t} u=c_{10} v+3 c_{12} u+8 \epsilon(u v) u-2 \epsilon u_{x x}, \\
-\partial_{t} v=-c_{10} u-3 c_{12} v-8 \epsilon(u v) v+2 \epsilon v_{x x} .
\end{gathered}
$$


The second pair can be understood as the complex conjugate of the first pair of equations, once we replace $t \rightarrow i t$ and $x \rightarrow i x$ and set $v=\bar{u}$. Moreover, once we choose $c_{10}=c_{12}=0$, these equations are reduced to the NLSE (2), i.e., i $\psi_{t}+\alpha_{2} S_{2}=0$ with $\alpha_{2}=2 \epsilon$.

\section{Level 3}

On level 3, we obtain the SSE, which has been discussed already in the end of Sec. III

$$
\begin{aligned}
& \partial_{t} u=\epsilon\left[-3 u\left(3 v u_{x}+u v_{x}\right)+u_{x x x}\right], \\
& \partial_{t} v=\epsilon\left[-3 v\left(3 u v_{x}+v u_{x}\right)+v_{x x x}\right] .
\end{aligned}
$$

\section{Level 4}

On level 4 , we obtain the set of $4^{\text {th }}$ order equations

$$
\begin{aligned}
& \partial_{t} u=c_{18} v+3 c_{20} u+6 \epsilon u u_{x} v_{x}+7 \epsilon u_{x x} u v+4 \epsilon\left(u_{x}\right)^{2} v+3 \epsilon u^{2} v_{x x}-12 \epsilon(u v)^{2} u-\frac{\epsilon}{2} u_{x x x x}, \\
& \partial_{t} v=-c_{18} u-3 c_{20} v-6 \epsilon v u_{x} v_{x}-7 \epsilon u v v_{x x}-4 \epsilon u\left(v_{x}\right)^{2}-3 \epsilon u_{x x} v^{2}+12 \epsilon(u v)^{2} v+\frac{\epsilon}{2} v_{x x x x}, \\
& -\partial_{t} u=c_{18} v+3 c_{20} u+6 \epsilon u u_{x} v_{x}+7 \epsilon u_{x x} u v+4 \epsilon\left(u_{x}\right)^{2} v+3 \epsilon u^{2} v_{x x}-12 \epsilon(u v)^{2} u-\frac{\epsilon}{2} u_{x x x x}, \\
& -\partial_{t} v=-c_{18} u-3 c_{20} v-6 \epsilon v u_{x} v_{x}-7 \epsilon u v v_{x x}-4 \epsilon u\left(v_{x}\right)^{2}-3 \epsilon u_{x x} v^{2}+12 \epsilon(u v)^{2} v+\frac{\epsilon}{2} v_{x x x x} .
\end{aligned}
$$

The second pair can be understood as the complex conjugate of the first pair of equations, once we replace $t \rightarrow i t$ and $x \rightarrow i x$ and set $v=\bar{u}$.

For the higher-order equations in this hierarchy, we set $c_{18}=c_{20}=0$. The resulting equation for the $4^{\text {th }}$ level of the hierarchy is then

$$
\partial_{t} \psi=-i \epsilon\left[6 \psi\left|\psi_{x}\right|^{2}+7|\psi|^{2} \psi_{x x}+4\left(\psi_{x}\right)^{2} \bar{\psi}+3 \psi^{2} \bar{\psi}_{x x}+12|\psi|^{4} \psi+\frac{1}{2} \psi_{x x x x}\right],
$$

where we replaced $u$ by $\psi$. Taking $\epsilon=-2 \alpha_{4}$, this provides Eq. (5), i.e., $i \psi_{t}+\alpha_{4} S_{4}=0$.

\section{E. Level 5}

On level 5, we obtain the set of $5^{\text {th }}$ order equations

$$
\begin{aligned}
& \partial_{t} u=\frac{1}{4} \epsilon\left(u_{x x x x x}+80(u v)^{2} u_{x}-5 u^{2} v_{x x x}-25 u\left(v_{x} u_{x}\right)_{x}+40 u^{3} v v_{x}-20 u_{x}^{2} v_{x}-15 u v u_{x x x}-30 v u_{x} u_{x x}\right), \\
& \partial_{t} v=\frac{1}{4} \epsilon\left(v_{x x x x x}+80(u v)^{2} v_{x}-5 v^{2} u_{x x x}-25 v\left(v_{x} u_{x}\right)_{x}+40 u v^{3} u_{x}-20 u_{x} v_{x}^{2}-15 u v v_{x x x}-30 u v_{x} v_{x x}\right) .
\end{aligned}
$$

Again, if we replace $t \rightarrow i t$ and $x \rightarrow i x$ and set $v=\bar{u}$, we obtain

$$
\begin{aligned}
\partial_{t} u & =\frac{1}{4} \epsilon\left(80|u|^{4} u_{x}+5 u^{2} v_{x x x}+25 u\left|u_{x}\right|_{x}^{2}+40|u|^{2} u^{2} v_{x}+20\left|u_{x}\right|^{2} u_{x}+15|u|^{2} u_{x x x}+30 v u_{x} u_{x x}+u_{x x x x x}\right), \\
\partial_{t} v & =\frac{1}{4} \epsilon\left(80|u|^{4} v_{x}+5 v^{2} u_{x x x}+25 v\left|u_{x}\right|_{x}^{2}+40|u|^{2} v^{2} u_{x}+20\left|u_{x}\right|^{2} v_{x}+15|u|^{2} v_{x x x}+30 u v_{x} v_{x x}+v_{x x x x x}\right),
\end{aligned}
$$

which finally reduces to the equation for the $5^{\text {th }}$ level of the SSE hierarchy

$$
\partial_{t} \psi=\frac{1}{4} \epsilon\left(\psi_{x x x x x}+80|\psi|^{4} \psi_{x}+5 \psi^{2} \bar{\psi}_{x x x}+25\left(\left|\psi_{x}\right|^{2}\right)_{x} \psi+40|\psi|^{2} \psi^{2} \bar{\psi}_{x}+20\left|\psi_{x}\right|^{2} \psi_{x}+15|\psi|^{2} \psi_{x x x}+30 \bar{\psi} \psi_{x} \psi_{x x}\right),
$$

where we replaced $u$ by $\psi$. Taking $\epsilon=4 \alpha_{5}$, this provides Eq. (6), i.e., $\psi_{t}-\alpha_{5} S_{5}=0$.

\section{F. Level 6}

On level 6, we obtain the set of $6^{\text {th }}$ order equations

$$
\begin{aligned}
\partial_{t} u= & \frac{\epsilon}{8}\left(-u_{x x x x x}+8 u^{2} v_{x x x x}+20 u v u_{x x x x}+37 u v_{x} u_{x x x}+32 u u_{x} v_{x x x}+43 v u_{x} u_{x x x}-170(u v)^{2} u_{x x}+53 u u_{x x} v_{x x}\right. \\
& \left.+45 u_{x}^{2} v_{x x}+31 v u_{x x}^{2}+95 u_{x} v_{x} u_{x x}-110 u^{3} v v_{x x}-330 u^{2} v u_{x} v_{x}-175 u v^{2} u_{x}^{2}-55 u^{3} v_{x}^{2}+160 u^{4} v^{3}\right),
\end{aligned}
$$




$$
\begin{aligned}
\partial_{t} v= & -\frac{\epsilon}{8}\left(-v_{x x x x x}+8 v^{2} u_{x x x x}+20 u v v_{x x x x}+37 v u_{x} v_{x x x}+32 v u_{x x x} v_{x}+43 u v_{x} v_{x x x}-170(u v)^{2} v_{x x}+53 v u_{x x} v_{x x}+45 v_{x}^{2} u_{x x}\right. \\
& \left.+31 u v_{x x}^{2}+95 u_{x} v_{x} v_{x x}-110 u u_{x x} v^{3}-330 u u_{x} v_{x} v^{2}-175 u^{2} v v_{x}^{2}-55 u_{x}^{2} v^{3}+160 u^{3} v^{4}\right) .
\end{aligned}
$$

If we replace, as before, $t \rightarrow i t$ and $x \rightarrow i x$ and set $v=\bar{u}$, we obtain

$$
\begin{aligned}
\partial_{t} u= & -i \frac{\epsilon}{8}\left(u_{x x x x x}+8 u^{2} v_{x x x x}+20|u|^{2} u_{x x x x}+37 u v_{x} u_{x x x}+32 u u_{x} v_{x x x}+43 v u_{x} u_{x x x}+170|u|^{4} u_{x x}+53 u\left|u_{x x}\right|^{2}+45 u_{x}^{2} v_{x x}\right. \\
& \left.+31 v u_{x x}^{2}+95\left|u_{x}\right|^{2} u_{x x}+110|u|^{2} u^{2} v_{x x}+330|u|^{2} u\left|u_{x}\right|^{2}+175|u|^{2} v u_{x}^{2}+55 u^{3} v_{x}^{2}+160|u|^{6} u\right), \\
\partial_{t} v= & i \frac{\epsilon}{8}\left(v_{x x x x x x}+8 v^{2} u_{x x x x}+20|u|^{2} v_{x x x x}+37 v u_{x} v_{x x x}+32 v v_{x} u_{x x x}+43 u v_{x} v_{x x x}+170|u|^{4} v_{x x}+53 v\left|u_{x x}\right|^{2}+45 v_{x}^{2} u_{x x}\right. \\
& \left.+31 u v_{x x}^{2}+95\left|u_{x}\right|^{2} v_{x x}+110|u|^{2} v^{2} u_{x x}+330|u|^{2} v\left|u_{x}\right|^{2}+175|u|^{2} u v_{x}^{2}+55 v^{3} u_{x}^{2}+160|u|^{6} u\right) .
\end{aligned}
$$

Apparently, the $2^{\text {nd }}$ equation is the complex conjugate of the first one, such that we finally obtain the equation for the $6^{\text {th }}$ level of the SSE hierarchy

$$
\begin{aligned}
\partial_{t} \psi= & -i \frac{\epsilon}{8}\left(\psi_{x x x x x}+8 \psi^{2} \bar{\psi}_{x x x x}+20|\psi|^{2} \psi_{x x x x}+37 \psi \bar{\psi}_{x} \psi_{x x x}+32 \psi \psi_{x} \bar{\psi}_{x x x}+43 \bar{\psi} \psi_{x} \psi_{x x x}+170|\psi|^{4} \psi_{x x}+53 \psi\left|\psi_{x x}\right|^{2}\right. \\
& \left.+45 \psi_{x}^{2} \bar{\psi}_{x x}+31 \bar{\psi} \psi_{x x}^{2}+95\left|\psi_{x}\right|^{2} \psi_{x x}+110|\psi|^{2} \psi^{2} \bar{\psi}_{x x}+330|\psi|^{2} \psi\left|\psi_{x}\right|^{2}+175|\psi|^{2} \bar{\psi} \psi_{x}^{2}+55 \psi^{3} \bar{\psi}_{x}^{2}+160|\psi|^{6} \psi\right),
\end{aligned}
$$

where $\psi=u$. Taking $\epsilon=-8 \alpha_{6}$, this provides Eq. (7), i.e., $i \psi_{t}+\alpha_{6} S_{6}=0$.

This process can be continued indefinitely, producing at each step the next functional of the SSE hierarchy. We restrict ourselves to giving the explicit expressions for the first 6 orders, as further extensions become increasingly cumbersome, although the whole procedure is straightforward.

\section{BASIC SOLUTIONS}

Finding solutions of the SSE is a complex task which has not been completely resolved, although the problem has been addressed in many previous works. ${ }^{37-43}$ This is related to both soliton and rogue wave solutions. Naturally, solving the SSE hierarchy is a much more involved issue. This will require future efforts of many researchers. However, the simplest solutions can be found with relative ease. Some examples are presented below.

\section{A. Basic "sech" solutions for hierarchy containing odd terms only}

Since the coefficients $\alpha_{2 n+1}$ are arbitrary, we can set the coefficient of the highest derivative to be unity in the derivations, and we still have an arbitrary real scaling factor on $x$. We consider real functions to start with and thus deal basically with the mKdV hierarchy. So

$$
u_{t}-\sum_{n=1}^{\infty}\left(\alpha_{2 n+1} S_{2 n+1}\right)=0 .
$$

Taking $u$ to be real in Eq. (3) gives

$$
u_{t}-\alpha_{3}\left(u_{x x x}+12 u^{2} u_{x}\right)=0 .
$$

The basic soliton solution of the SSE (54) is

$$
u=\frac{g}{\sqrt{2}} \operatorname{sech}\left[g\left(x+\alpha_{3} g^{2} t\right)\right]
$$

Here, $\alpha_{3} g^{2}$ represents a velocity. The solution (55) is real, and in this case the SSE and its basic solution are the same as the "standard" $\mathrm{mKdV}, u_{t}-a_{3}\left(u_{x x x}+6 u^{2} u_{x}\right)=0$, apart from a scaling of $\sqrt{2}$ on $x$. We expect this form to be valid for all odd-numbered functionals in the set. If $u(x, t)$ is an mKdV solution, then overall scaling shows that $u^{\prime}=$ $q u\left(q x, q^{3} t\right)$ is also a solution for real $q$, so we could just set $g=1$ in the above, without loss of generality. For a general odd order equation, we have

$$
u_{2 n+1}=\frac{g}{\sqrt{2}} \operatorname{sech}\left[g\left(x+\alpha_{2 n+1} g^{2 n} t\right)\right]
$$

for $n=1,2,3, \ldots$ Thus, the scaling, $x \rightarrow g x$ and $\alpha_{2 n+1} t \rightarrow \alpha_{2 n+1} g^{2 n+1} t$, with overall multiplication by $g$, is similar to that for the NLSE hierarchy. ${ }^{34}$

\section{B. Polynomial and periodic solutions}

The basic polynomial solution of $u_{t}-\alpha_{3} S_{3}=0$, with background set to -1 , is

$$
u_{3}=\frac{4}{1+8\left(x+12 \alpha_{3} t\right)^{2}}-1 .
$$

The periodic solution is

$$
u_{3}=\frac{k^{2}}{2-\sqrt{4-k^{2}} \cos \left[\sqrt{2} k\left(v_{3} t+x\right)\right]}-1,
$$

where $v_{3}=-2 \alpha_{3}\left(k^{2}-6\right)$ and $|k|<2$. 
The $5^{\text {th }}$ order equation will have solutions of the same form with different coefficients. We now consider

$$
u_{t}-\alpha_{5} S_{5}=0
$$

from Eq. (6) with real $\psi=u$. We get

$$
S_{5}=u_{x x x x x}+20 u_{x x x} u^{2}+120 u_{x} u^{4}+80 u u_{x} u_{x x}+20 u_{x}^{3},
$$

which agrees with the real solutions of Eq. (52). This is the same as the first higher order $\mathrm{mKdV}$ equation. ${ }^{48}$ This applies for all odd order equations and clarifies the fact that the SSE hierarchy is a form of "complexification" of the $\mathrm{mKdV}$ hierarchy.

The basic polynomial solution, with background set to -1 , is

$$
u_{5}=\frac{4}{1+8\left(x+120 \alpha_{5} t\right)^{2}}-1,
$$

while the periodic solution is

$$
u_{5}=\frac{k^{2}}{2-\sqrt{4-k^{2}} \cos \left[\sqrt{2} k\left(v_{5} t+x\right)\right]}-1,
$$

where $v_{5}=4 \alpha_{5}\left(k^{4}-10 k^{2}+30\right)$ and $|k|<2$.

The $7^{\text {th }}$ order functional is

$$
\begin{aligned}
S_{7}= & u_{x x x x x x x}+28 u\left(u_{x x x x x} u+2 u_{x} u_{x x x x}\right) \\
& +112\left[u u_{x} u_{x x x x}+\left(u u_{x x}+u_{x}^{2}\right) u_{x x x}\right]+84 u_{x x}\left(2 u u_{x x x}+u_{x} u_{x x}\right) \\
& +140 u_{x}\left(u_{x} u_{x x x}+2 u_{x x}^{2}\right)+280 u^{3}\left(u u_{x x x}+40 u_{x} u_{x x}\right) \\
& +560 u_{x} u^{2}\left(2 u u_{x x}+3 u_{x}^{2}\right)+1120 u^{6} u_{x} .
\end{aligned}
$$

The equation of $7^{\text {th }}$ order has solutions of the same form as above but with different coefficients. Instead of presenting it, let us turn our attention to the general case.

\section{Solutions of composite equations with many functionals}

We can now include more than one functional at a time. Namely, for the equation

$$
u_{t}-\alpha_{3} S_{3}-\alpha_{5} S_{5}-\alpha_{7} S_{7}-\cdots=0 \text {, }
$$

we have the soliton solution

$$
u_{s}=\frac{g}{\sqrt{2}} \operatorname{sech}[g(x+s t)]
$$

with $s=\sum_{n=1}^{\infty} \alpha_{2 n+1} g^{2 n}$, and the polynomial solution

$$
u_{\text {poly }}=\frac{4}{1+8\left(x+v_{a} t\right)^{2}}-1
$$

with $v_{a}=4\left(3 \alpha_{3}+30 \alpha_{5}+280 \alpha_{7}+2520 \alpha_{9}+\cdots\right)$. For the infinite hierarchy

$$
v_{a}=\sum_{n=1}^{\infty} \frac{2^{n}}{(n !)^{2}}(2 n+1) ! \alpha_{2 n+1} .
$$

The periodic solution in this case is

$$
u_{\mathrm{per}}=\frac{k^{2}}{2-\sqrt{4-k^{2}} \cos \left[\sqrt{2} k\left(v_{\mathrm{per}} t+x\right)\right]}-1 \text {, }
$$

where

$$
\begin{aligned}
v_{\text {per }}= & -2 \alpha_{3}\left(k^{2}-6\right)+4 \alpha_{5}\left(k^{4}-10 k^{2}+30\right) \\
& -8 \alpha_{7}\left(k^{6}-14 k^{4}+70 k^{2}-140\right) \\
& +16 \alpha_{9}\left(k^{8}-18 k^{6}+126 k^{4}-420 k^{2}+630\right)-\cdots,
\end{aligned}
$$

with $|k|<2$. Again, these solutions apply to the equations up to infinite order, with just the velocity coefficients changing. These coefficients can be written as a summation of the form $\sum_{n=1}^{\infty}\left(\alpha_{2 n+1} c_{n}\right)$. Thus, for the infinite hierarchy

$$
v_{\mathrm{per}}=\sum_{n=1}^{\infty} \frac{2^{n}(2 n+1) !}{(n !)^{2}} \alpha_{2 n+1}{ }_{2} F_{1}\left(1,-n ; \frac{3}{2} ; \frac{k^{2}}{4}\right),
$$

where ${ }_{2} F_{1}(\ldots)$ is the hypergeometric function.

\section{Rogue wave solutions}

We have recently shown that the mKdV equation has rogue wave solutions with parameters similar to the rogue wave solutions of the NLSE. ${ }^{49}$ For each of these solutions, the central rogue wave has the same amplitude as that of the corresponding rogue-wave of the NLSE, but it is located on top of a soliton. The central part generally has a close resemblance to the shape of an NLSE rogue wave.

Solutions with similar features can be derived for the SSE. Namely,

$$
u_{3}^{\mathrm{rw}}=1+12 \frac{N_{3}}{D_{3}},
$$

where

$$
N_{3}=3-16\left(x-12 \alpha_{3} t\right)\left[4\left(x-12 \alpha_{3} t\right)^{3}+3\left(x-44 \alpha_{3} t\right)\right],
$$

and

$$
\begin{aligned}
D_{3}= & 512 x^{6}+192 x^{4}+216 x^{2}+9-192 \alpha_{3} t\left(192 x^{4}-16 x^{2}+51\right) x \\
& +2\left(24 \alpha_{3} t\right)^{2}\left(960 x^{4}-240 x^{2}+139\right)-32\left(24 \alpha_{3} t\right)^{3} \\
& \times\left(40 x^{2}-9\right) x+4\left(24 \alpha_{3} t\right)^{4}\left(120 x^{2}-13\right) \\
& +3\left(48 \alpha_{3} t\right)^{5}\left(2 \alpha_{3} t-x\right) .
\end{aligned}
$$

The central amplitude is obviously 5, matching the amplitude of the second order NLSE rogue wave. Here, we restrict ourselves to this example, although it can be extended to higher-order solutions of this equation as well as to those that cover the higher order equations of the SSE hierarchy.

\section{EARLIER PAINLEVÉ RESULTS}

The Sasa-Satsuma hierarchy has been missed in the previous studies for a relatively simple reason. Painlevé analysis for fourth order equations was given earlier in Ref. 35. These equations take the form 


$$
i u_{t}+K_{2}+\gamma_{1} L_{4}=0,
$$

where $\gamma_{1}$ is a constant, $K_{2}$ is the nonlinear Schrödinger functional, and $L_{4}$ is similar to $K_{4}$ in Ref. 34

$$
K_{4}=2 \bar{u}_{x x} u^{2}+6 u|u|^{4}+4\left|u_{x}\right|^{2} u+8|u|^{2} u_{x x}+6 \bar{u} u_{x}^{2}+u_{x x x x},
$$

apart from the point that the fixed coefficients are replaced by 5 parameters. This would appear to include the functional $S_{4}$ in Eq. (5), but it does not, because the authors have imposed physical constraints (to study the Heisenberg spin magnet) on the parameters, so that each of the 5 parameters is written in terms of a single parameter, viz., $\frac{\gamma_{2}}{\gamma_{1}}$. The Painlevé singularity structure analysis carried out then shows that the only value giving integrability is $\frac{\gamma_{2}}{\gamma_{1}}=-\frac{5}{2}$, corresponding to $K_{4}$ above. Indeed, $i u_{t}+\alpha_{2} K_{2}+\alpha_{4} K_{4}=0$ is usually called the LPD equation, whether $\alpha_{2}$ is zero or not. Beyond the basic NLS itself, it forms the first even higherorder equation in the NLS hierarchy. However, even allowing for a scaling factor $a$, there is no combination of $\left\{a, \frac{\gamma_{2}}{\gamma_{1}}\right\}$ which allows Eq. (5) to be represented in the form used in Ref. 35, and so it is not included in the set studied there. As such, the existence of the integrable equation $i u_{t}+\alpha_{4} S_{4}=0$, with $S_{4}$ from Eq. (5), is not contradicted by singularity analysis.

\section{CONCLUSIONS}

In this work, we have found the infinite Sasa-Satsuma hierarchy of integrable equations. This new hierarchy significantly expands the family of integrable equations related to the NLSE. We have no doubt that this set is not less important than the infinite NLSE hierarchy presented earlier. It was missing from the previous works for the reasons explained in Sec. VI. Clearly, every single addition of a physically relevant integrable equation to the collection of known cases significantly enriches our ability to mathematically describe complicated evolution problems. Our new hierarchy contains an infinite number of equations with free variable parameters. We stress that the parameters do not need to be small, whereas they must be small for perturbation techniques. They are finite, and their presence allows that these equations can be adjusted to practical applications as closely as possible.

Moreover, we have presented the lowest-order operators of this hierarchy in explicit form, allowing us to deal with individual equations of the hierarchy using relatively simple common tools. Explicit forms simplify the handling of these equations without the excessive complexities of other mathematical formulations.

Of course, solving these equations is another matter. Finding solutions of these equations is a difficult task. As the original Sasa-Satsuma equation has much more involved solutions than the NLSE, the solutions of this hierarchy can also be highly complicated. Nevertheless, some solutions of the whole infinite hierarchy can even be written in explicit form. We provided a few simple examples demonstrating this. More complex solutions need more effort to derive, but the task can be done due to the integrability of the hierarchy.

We have also shown that this hierarchy is closely related to the hierarchy of $\mathrm{mKdV}$ equations. This way, the interconnections between various cases can be established. One clear advantage of having a new hierarchy is the following. Having integrable cases as reference equations, we can construct approximations around them, thus significantly increasing the set of cases that can be treated analytically. Even if some particular equation does not describe a certain application accurately, it can be made close to it using approximations around the integrable case. Thus, step-by-step, more practical problems of interest can be solved using the closest integrable equation and its approximations.

To conclude, we believe that the hierarchy of equations that we have found here is not the last one. Even more involved hierarchies based on NLSE may be constructed in future. This is just a question of time.

\section{ACKNOWLEDGMENTS}

The authors gratefully acknowledge the support of the Australian Research Council (Discovery Projects DP140100265 and DP150102057) and support from the Volkswagen Stiftung. N.A. is a recipient of the Alexander von Humboldt Award. U.B. acknowledges support by the German Research Foundation in the framework of the Collaborative Research Center 787 "Semiconductor Nanophotonics" under project B5. Sh.A. acknowledges support of the German Research Foundation under Project No. 389251150.

${ }^{1}$ A. Hasegawa and F. Tappert, "Transmission of stationary nonlinear optical pulses in dispersive dielectric fibers. I. Anomalous dispersion," Appl. Phys. Lett. 23, 142 (1973).

${ }^{2}$ V. E. Zakharov, "Stability of periodic waves of finite amplitude on a surface of deep fluid,” J. Appl. Mech. Tech. Phys. 9, 190-194 (1968).

${ }^{3}$ H. C. Yuen and B. M. Lake, "Nonlinear dynamics of deep-water gravity waves,” Adv. Appl. Mech. 22, 67-228 (1982).

${ }^{4}$ D. J. Benney and A. C. Newell, "The propagation of nonlinear wave envelopes," J. Math. Phys. 46, 133-139 (1967).

${ }^{5}$ V. E. Zakharov and A. B. Shabat, "Exact theory of two-dimensional selffocussing and one-dimensional self-modulation of waves in nonlinear media," Sov. Phys. - JETP 34, 62-69 (1972).

${ }^{6}$ V. S. Gerdjikov, I. M. Uzunov, E. G. Evstatiev, and G. L. Diankov, "Nonlinear Schrödinger equation and N-soliton interactions," Phys. Rev. E 55, 6039 (1997).

${ }^{7}$ N. Akhmediev and V. I. Korneev, "Modulation instability and periodic solutions of the nonlinear Schrödinger equation," Theor. Math. Phys. (USSR) 69, 1089 (1986) [Teor. Mat. Fiz. 69, 189-194 (1986)].

${ }^{8}$ N. Vishnu Priya, M. Senthilvelan, and M. Lakshmanan, “ABs, Ma solitons, and general breathers from rogue waves: A case study in the Manakov system," Phys. Rev. E 88, 022918 (2013).

${ }^{9}$ F. Baronio, "ABs and Peregrine solitary waves in a quadratic medium," Opt. Lett. 42, 1756 (2017).

${ }^{10}$ V. A. Makarov and V. M. Petnikova, "The distinctive feature of long time adiabatic modulation in the context of cnoidal wave and AB interaction," Laser Phys. 27, 025402 (2017).

${ }^{11}$ B. Kibler, J. Fatome, C. Finot, G. Millot, F. Dias, G. Genty, N. Akhmediev, and J. M. Dudley, "The Peregrine soliton in nonlinear fibre optics," Nat. Phys. 6, 790 (2010).

${ }^{12}$ J. M. Dudley, G. Genty, and S. Coen, "Supercontinuum generation in photonic crystal fiber,” Rev. Mod. Phys. 78, 1135 (2006).

${ }^{13}$ A. Mussot, A. Kudlinski, M. Droques, P. Szriftgiser, and N. Akhmediev, "Fermi-Pasta-Ulam recurrence in nonlinear fiber optics: The role of reversible and irreversible losses," Phys. Rev. X 4, 011054 (2014). 
${ }^{14}$ V. B. Bobrov and S. A. Trigger, "Bose-Einstein condensate wave function and nonlinear Schrödinger equation,” Bull. Lebedev Phys. Inst. 43, 266 (2016).

${ }^{15}$ V. S. Bagnato, D. J. Frantzeskakis, P. G. Kevrekidis, B. A. Malomed, and D. Mihalache, "Bose-Einstein condensation: Twenty years after," Rom. Rep. Phys. 67, 5-20 (2015).

${ }^{16}$ B. Malomed, L. Torner, F. Wise, and D. Mihalache, "On multidimensional solitons and their legacy in contemporary atomic, molecular and optical physics," J. Phys. B: At., Mol. Opt. Phys. 49, 170502 (2016).

${ }^{17}$ N. Akhmediev, A. Ankiewicz, and M. Taki, "Waves that appear from nowhere and disappear without a trace," Phys. Lett. A 373, 675-678 (2009).

${ }^{18}$ F. Baronio, M. Conforti, A. Degasperis, and S. Lombardo, "Rogue waves emerging from the resonant interaction of three waves," Phys. Rev. Lett. 111, 114101 (2013).

${ }^{19}$ F. Baronio, A. Degasperis, M. Conforti, and S. Wabnitz, "Solutions of the vector nonlinear Schrödinger equations: Evidence for deterministic rogue waves," Phys. Rev. Lett. 109, 044102 (2012).

${ }^{20}$ S. Chen, F. Baronio, J. M. Soto-Crespo, P. Grelu, and D. Mihalache, "Versatile rogue waves in scalar, vector, and multidimensional nonlinear systems," J. Phys. A: Math. Theor. 50, 463001 (2017).

${ }^{21}$ D. Mihalache, "Multidimensional localized structures in optical and matter-wave media: A topical survey of recent literature," Rom. Rep. Phys. 69, 403 (2017).

${ }^{22}$ A. Ankiewicz and N. Akhmediev, "Multi-rogue waves and triangular numbers," Rom. Rep. Phys. 69, 104 (2017).

${ }^{23}$ K. B. Dysthe, "Note on a modification to the nonlinear Schrödinger equation for application to deep water waves," Proc. R. Soc. London, Ser. A 369, 105-114 (1979).

${ }^{24}$ S. B. Cavalcanti, J. C. Cressoni, H. R. da Cruz, and A. S. Gouveia-Neto, "Modulation instability in the region of minimum group-velocity dispersion of single-mode optical fibers via an extended nonlinear Schrödinger equation," Phys. Rev. A 43, 6162-6165 (1991).

${ }^{25} \mathrm{M}$. Trippenbach and Y. B. Band, "Effects of self-steepening and selffrequency shifting on short-pulse splitting in dispersive nonlinear media," Phys. Rev. A 57, 4791 (1991).

${ }^{26}$ Yu. V. Sedletskii, "The fourth-order nonlinear Schrödinger equation for the envelope of Stokes waves on the surface of a finite-depth fluid," Sov. Phys. - JETP 97, 180-193 (2003) [Zh. Eksp. Teor. Fiz. 124, 200 (2003)].

${ }^{27}$ A. V. Slunyaev, "A high-order nonlinear envelope equation for gravity waves in finite-depth water," Sov. Phys. - JETP 101(5), 926-941 (2005) [Zh. Eksp. Teor. Fiz. 128, 1061-1077 (2005)].

${ }^{28}$ M. J. Potasek, "Exact solutions for an extended nonlinear Schrödinger equation," Phys. Lett. A 154, 449-452 (1991).

${ }^{29}$ R. Hirota, "Exact envelope-soliton solutions of a nonlinear wave equation," J. Math. Phys. 14, 805 (1973).

${ }^{30}$ N. Sasa and J. Satsuma, "New-type of soliton solutions for a higher-order nonlinear Schrödinger equation,” J. Phys. Soc. Jpn. 60, 409-417 (1991).

${ }^{31}$ M. Lakshmanan, K. Porsezian, and M. Daniel, "Effect of discreteness on the continuum limit of the Heisenberg spin chain," Phys. Lett. A 133, 483-488 (1988).

${ }^{32}$ S. M. Hoseini and T. R. Marchant, "Solitary wave interaction and evolution for a higher-order Hirota equation," Wave Motion 44, 92 (2006).
${ }^{33}$ D. J. Kedziora, A. Ankiewicz, A. Chowdury, and N. Akhmediev, "Integrable equations of the infinite nonlinear Schrödinger equation hierarchy with time variable coefficients," Chaos 25, 103114 (2015).

${ }^{34}$ A. Ankiewicz, D. J. Kedziora, A. Chowdury, U. Bandelow, and N. Akhmediev, "Infinite hierarchy of nonlinear Schrödinger equations and their solutions," Phys. Rev. E 93, 012206 (2016).

${ }^{35}$ K. Porsezian, M. Daniel, and M. Lakshmanan, "On the integrability aspects of the one-dimensional classical continuum isotropic biquadratic Heisenberg spin chain,” J. Math. Phys. 33, 1807-1816 (1992).

${ }^{36}$ A. Ankiewicz, J. M. Soto-Crespo, M. A. Chowdhury, and N. Akhmediev, "Rogue waves in optical fibers in presence of third-order dispersion, self-steepening, and self-frequency shift," J. Opt. Soc. Am. B 30, 87 (2013).

${ }^{37}$ D. Mihalache, L. Torner, F. Moldoveanu, N. C. Panoiu, and N. Truta, "Soliton solutions for a perturbed nonlinear Schrödinger equation," J. Phys. A: Math. Gen. 26, L757-L765 (1993).

${ }^{38}$ D. Mihalache, N. C. Panoiu, F. Moldoveanu, and D.-M. Baboiu, "The Riemann problem method for solving a perturbed nonlinear Schrodinger equation describing pulse propagation in optical fibres," J. Phys. A: Math. Gen. 27, 6177-6189 (1994).

${ }^{39}$ D. Mihalache, L. Torner, F. Moldoveanu, N. C. Panoiu, and N. Truta, "Inverse-scattering approach to femtosecond solitons in monomode optical fibers,” Phys. Rev. E 48, 4699 (1993).

${ }^{40}$ O. C. Wright III, "Sasa - Satsuma equation, unstable plane waves and heteroclinic connections," Chaos, Solitons Fractals 33, 374-387 (2007).

${ }^{41}$ C. Gilson, J. Hientarinta, J. Nimmo, and Y. Ohta, "Sasa - Satsuma higherorder nonlinear Schrödinger equation and its bilinearization and multisoliton solutions," Phys. Rev. E 68, 016614 (2003).

${ }^{42}$ J. Kim, Q.-H. Park, and H. J. Shin, "Conservation laws in higher-order nonlinear Schrödinger equations," Phys. Rev. E 58, 6746-6751 (1998).

${ }^{43} \mathrm{U}$. Bandelow and N. Akhmediev, "Persistence of rogue waves in extended nonlinear Schrödinger equations: Integrable Sasa-Satsuma case," Phys. Lett. A 376, 1558-1561 (2012).

${ }^{44}$ J. M. Soto-Crespo, N. Akhmediev, N. Devine, and N. P. Hoffmann, "Rogue waves of the Sasa-Satsuma equation in a chaotic wave field," Phys. Rev. E 90, 032902 (2014).

${ }^{45}$ S. V. Manakov, "On the theory of two-dimensional stationary selffocusing of electromagnetic waves," Sov. Phys. - JETP 38, 248-253 (1974) [Zh. Eksp. Teor. Fiz. 65, 505-516 (1973)].

${ }^{46} \mathrm{~L}$. Wu, G. He, and X. Geng, "The full positive flows of Manakov hierarchy, Hamiltonian structures and conservation laws," Appl. Math. Comput. 220, 20-37 (2013).

${ }^{47} \mathrm{G}$. Tu, "The trace identity, a powerful tool for constructing the Hamiltonian structure of integrable systems," J. Math. Phys. 30, 330-338 (1989).

${ }^{48}$ Y. Matsuno, "Bilinearization of nonlinear evolution equations. II. Higherorder modified Korteweg-de Vries equations," J. Phys. Soc. Jpn. 49, 787-794 (1980).

${ }^{49}$ A. Ankiewicz and N. Akhmediev, "Rogue wave-type solutions of the $\mathrm{mKdV}$ equation and their relation to known NLSE rogue wave solutions," Nonlinear Dyn. 91, 1931 (2018). 